\title{
Medico-Legal Aspects of Using Tissue Plasminogen Activator in Acute Ischemic Stroke
}

Nhu T. Bruce, MD

William P. Neil, MD

Justin A. Zivin, MD, PhD*

\author{
Address \\ *San Diego VA Healthcare System, Neurology Service (127), 3350 La Jolla \\ Village Drive, San Diego, CA 92161, USA \\ Email: jzivin@ucsd.edu \\ Published online: 22 March 2011 \\ (C) The Author(s) 2011. This article is published with open access at Springerlink.com
}

\section{Opinion statement}

Intravenous alteplase or tissue plasminogen activator (tPA) has been the standard of care with proven efficacy for acute ischemic stroke for over a decade. Despite this, only a small fraction of potentially eligible stroke patients receive this medication. There seems to be a fear among practitioners of legal repercussions as a result of an increased risk of intracerebral hemorrhage due to tPA. This review of legal cases involving tPA will show that instead, physicians are often found liable as a result of not treating with tPA.

\section{Introduction}

Intravenous tissue plasminogen activator (tPA) has been approved by the US Food and Drug Administration (FDA) for the treatment of acute ischemic stroke within $3 \mathrm{~h}$ of symptom onset since 1996 . Despite multiple randomized controlled trials and statistical evidence supporting its efficacy over placebo, there is still some reluctance to use the drug because of the increased risk of hemorrhage $[1,2 \bullet]$. However, these risks have been highly exaggerated in the past and the true benefits have been grossly underestimated [2•]. Given the dissension among trained medical professionals and the variations in data interpretation, it is not surprising that there are current concerns over potential litigation involving the use and non-use of the drug.

\section{Pathophysiology}

Alteplase was the first recombinant tissue-type plasminogen activator that is identical to intrinsic human tPA. In the body, tissue-type plasminogen activator is synthesized and released by cells of the vascular endothelium and is responsible for most of the body's natural efforts to prevent excessive thrombus formation or dissolve those that are no longer needed [3].

Alteplase is fibrin specific, with a plasma half-life of 3 to 6 min and should hypothetically be effective only at the surface of a fibrin clot. However, despite greater fibrin affinity than any other thrombolytic agent, a general systemic lytic state can occur with the potential for systemic bleeding [4]. 


\section{Treatment}

The use of tPA in acute ischemic stroke

- IV tPA is the only FDA-approved therapy for acute ischemic stroke. Recombinant tPA was initially developed in the mid 1980s as a potential treatment for acute coronary artery occlusion. Studies on the use of thrombolytic therapy in acute strokes have been conducted since the 1950s [5]. Although other thrombolytic agents such as streptokinase have been complicated by increased rates of hemorrhage, they provided the basis for more sophisticated randomized trials [6].

- Given its greater fibrin specificity, recombinant tPA was favored in studies during the 1990s because of its safety as compared with other thrombolytic agents.

- Earlier work from past decades suggested better outcomes in patients who were treated soon after symptom onset $[7,8]$. Open-labeled dose-escalation studies showed that there was a statistical correlation between the incidence of symptomatic intracerebral hemorrhage (ICH) and total dose of drug administered [9].

- In 1995, the results of the National Institute of Neurological Disorders and Stroke (NINDS) stroke trial were published. This was a twopart, randomized, double-blind trial of intravenous (IV) tPA at a dose of $0.9 \mathrm{mg} / \mathrm{kg}$ versus placebo in patients with stroke symptoms within $3 \mathrm{~h}$ of onset. It showed that patients who received IV tPA within $3 \mathrm{~h}$ of their stroke onset were at least 30\% more likely to have little or no disability at 3 months, with an odds ratio for favorable outcome of 1.7 [10]. In an independent 2004 analysis of original data from this trial using an independent panel comprised of neurologists and emergency room physicians who were experts in the field of stroke to assess outcomes, Saver [11] showed that the average number needed to treat for one patient to have a better outcome, defined as a change in the modified Rankin score (mRS) of 1 or more, is 3.1. Therefore, as judged by these experts, for every 100 patients treated with tPA, 32 will have a better final outcome because of the treatment [11].

- The European Cooperative Acute Study (ECASS III) widened the potential window for treatment from $3 \mathrm{~h}$ to $4.5 \mathrm{~h}$ with their published findings in 2008 showing that there was still a more favorable outcome with patients who were treated with IV tPA compared to placebo with an odds ratio of 1.34 [12]. The patient selection was slightly different from the NINDS trials in that ECASS III excluded patients older than 80 years of age, had a combination of prior stroke and diabetes, and National Institute of Health Stroke Scale score greater than 25 [12]. Also, this trial independently confirmed in a placebo-controlled study that tPA is an effective treatment, a fact that was initially established by the original NINDS study [10]. 
- Although ECASS III showed some benefits to patients treated in the 3- to 4.5-h time period, patients who were treated closer to the time of stroke onset tended to have better outcomes, and it is still only FDA approved for use within $3 \mathrm{~h}$ of onset. In a 2010 pooled analysis of the eight major randomized placebo-controlled trials of tPA and acute ischemic stroke, investigators showed that the odds of a favorable outcome at 3 months decreased as the onset to time to treatment (OTT) number increased and the odds of mortality increased with the OTT. Adjusted odds of favorable outcomes in patients treated with tPA were 2.55 for patients treated from 0 to $90 \mathrm{~min}, 1.64$ for those between 91 to $180 \mathrm{~min}, 1.34$ for 181 to $270 \mathrm{~min}$ and 1.22 for 271 to $360 \mathrm{~min}$. Adjusted odds of mortality were, respectively, $0.78,1.13,1.22$, and 1.49 for the same time ranges, indicating that patients had an increased chance of better outcome, including survival, if they were treated early [13].

\section{Bleeding risks}

- Despite numerous studies and analyses showing the benefits of tPA and reviews of the legal literature suggesting that physicians are more liable to lawsuits for failure to use tPA, there is still reluctance among providers, with most citing the concern for bleeding risks [1]. The NINDS trials showed a risk of ICH of $6.4 \%$ in patients who were treated with tPA compared to $0.6 \%$ in the placebo group [10].

- In 2007, Saver [2•] again analyzed the original data from the NINDS stroke studies and found that when compared to placebo, the patients in the treatment group who experienced symptomatic sICH were older, had more severe stroke symptoms, higher serum glucose, and more likely to have mass effect on their pretreatment CT scan. The calculated number needed to harm for one patient to have a severely disabling or deadly outcome (defined as a mRS $>4$ ) due to their tPA-associated sICH was 126 , or approximately one of every 100 patients who experience sICH in the setting of tPA treatment [2•].

- In 1996, the FDA approved the use of tPA in acute ischemic stroke, and in August of 2000 the American Heart Association upgraded its recommendation from Class IIB (optional) to Class I (definite recommendation). Multiple national organizations, most of them comprised of emergency room physicians, have challenged this change, arguing that more evidence is needed before it could be the standard of care [14].

- Such variations in clinical practice suggest that, currently, there may be no universally accepted standard when it comes to the treatment of acute ischemic stroke. Given more studies proving the efficacy of tPA as an effective treatment in acute ischemic stroke and the emergence of stroke centers, this is rapidly changing. 
- In 2000, a prospective analysis of 29 Cleveland-area hospitals showed that only $2 \%$ of patients admitted for acute ischemic stroke from July of 1997 to June of 1998 received tPA. Seventeen percent of these patients presented within $3 \mathrm{~h}$ of symptom onset, and of these patients only $10.4 \%$ received treatment. The rate of sICH was $15.7 \%$, much higher than the NINDS study, but in half of the patients treated there was a deviation to the protocol established by the study [15]. Numbers similar to these have been reported in other studies, suggesting that the majority of patients who meet criteria for tPA treatment do not receive therapy. Katzan et al. [16] published an update a few years later after a stroke quality improvement program was implemented in nine of the Cleveland-area hospitals and showed $18.8 \%$ of patients who presented within $3 \mathrm{~h}$ received tPA treatment. The rate of sICH in these patients was $6.4 \%$, similar to the original NINDS study, and protocol violations decreased to $19 \%$ [16]. Although these numbers may still appear elevated, they imply that closer adherence to the original study criteria decreases the rate of adverse effects.

- Medico-legal concerns are a major barrier to treating stroke patients with tPA. A recent empirical study of trial court cases involving tPA and ischemic stroke showed that most cases involved emergency physicians, and that liability is most often associated with failure to treat with IV tPA, rather than adverse events associated with its use $[17 \bullet \bullet]$.

- The definition of medical malpractice is an act or omission by a health care provider that deviates or fails to conform to the accepted standards of practice in the medical community that subsequently results in injury to the patient. In order to prove such negligence and achieve a successful malpractice claim, a plaintiff has to establish the following four elements:

1. a duty was owed (an element that exists whenever a hospital or health care provider undertakes care or treatment of a patient)

2. a duty was breached (the provider failed to conform or deviated from the appropriate standard of care)

3. the breach caused an injury

4. the injury led to damages (the foundation for a suit, without which, there is no basis for a claim, regardless of whether the medical provider was negligent) [18].

- A 2006 review by Weintraub [19] depicted several anecdotal cases to illustrate the variation in types of malpractice claims, from failure to treat to adverse outcomes from treatment, and illustrated the difficulties of defining negligence without an accepted standard of care. He argued that given the continual evolution of accepted stroke therapy, the jury 
could not always distinguish medical malpractice from medical maloccurrence [19]. However, although anecdotes might give some insight to the trend in public perception, they are merely descriptive and do not offer measurable variables that could be use to establish any meaningful standard or code of conduct. Baumbauer et al. [1] hypothesized that the reason physicians may not administer IV tPA is their fear of potential lawsuits for medical malpractice. To evaluate their theory, they queried the LexisNexis Academic database for cases involving physicians and the use of tPA in stroke. Most cases ended with a settlement and, therefore, were not publicly available, and the database contains mostly appellate level decisions. However, they were able to identify seven cases with final decisions. In all cases, claims were filed for the failure to administer tPA, but only one of these cases favored the plaintiff [1].

- In a 2008 review of lawsuits involving tPA, Liang and Zivin [17••] identified 33 cases that involved tPA and stroke. More than half of these cases involved emergency room physicians and less than 20\% involved neurologists. In every case in which a neurologist was a defendant, the emergency physician was also named. In about two thirds of the cases, plaintiffs stated a failure or delay in diagnosis of stroke. In $87.9 \%$ of the cases, patients claimed there was a failure to treat with IV tPA, whereas only $9.1 \%$ cited that the use of the drug caused their injury. In 12 of the 33 cases, the plaintiffs were given positive verdicts, with 2 of the 12 rewarded for injury from the use of the drug and the majority for failure to treat with tPA. With the increasing use and expansion of the therapeutic time window, physicians are interested more than ever about the potential legal ramifications $[17 \bullet \bullet]$.

- In regard to failure to treat, a plaintiff has the burden of not only proving that there was a deviation from the standard of care, but also that the diversion caused direct injury. Various jurisdictions may rule differently on whether to use this standard, or they may require that tPA show benefit in more than $50 \%$ of patients without including the placebo responders. In a 2008 reanalysis of the NINDS data, Liang et al. [20 $]$ used proportionate pairs (one treated with placebo, the other with tPA) and placed patients in all possible combinations using the Wilcoxon test. From these pairs, they determined which of the two had better outcomes, described as a better mRS score at 90 days compared to baseline prior to treatment. In the case of a tie between patients, a larger change in the NIHSS was considered the superior outcome. They reported that the probability that IV tPA was superior was $57.3 \%$. Although this number may have included patients who would have improved spontaneously without tPA, it is reasonable to include them because it would be impossible for clinicians to identify these patients prior to treatment $[20 \bullet]$.

- Traditionally, legal causation is demonstrated by showing that there was a greater than $50 \%$ chance that the failure to give the drug caused the patient injury, but some courts will allow a plaintiff to recover for the loss of opportunity for a better outcome [21,22]. In a search of state and federal cases Thiess et al. [23] identified 20 trial court and six appellate 
cases that involved suits over the nonuse of IV tPA for patients with a stroke, and none for injury caused allegedly by the drug. In 14 of 20 cases, the verdict was for the defendant [23].

- The preponderance of evidence suggests that IV tPA is not only effective, but it is safe. Physicians caring for stroke patients should not deny its use in appropriate patients for fear of being sued. More often, it seems verdicts against the defendant arise in cases where IV TPA was not used or where there were deviations from the standard of care. However, the legal landscape is now rapidly changing and as more cases are adjudicated, the conclusions we make are quickly changing.

\section{Disclosure}

The authors report no potential conflicts of interest relevant to this article.

\section{Open Access}

This article is distributed under the terms of the Creative Commons Attribution Noncommercial License which permits any noncommercial use, distribution, and reproduction in any medium, provided the original author(s) and source are credited.

\section{References and Recommended Reading}

Papers of particular interest, published recently, have been

highlighted as:

- Of importance

- Of major importance

1. Bambauer KZ, Johnston SC, Bambauer DE, Zivin JA. Reasons why few patients with acute stroke receive tissue plasminogen activator. Arch Neurol. 2006;63:661-4.

2. Saver JL. Hemorrhage after thrombolytic therapy for stroke: The clinically relevant number needed to harm. Stroke. 2007;38:2279-83.

A re-analysis of the NINDS trial showed the clinically relevant number needed to harm for a patient to have a worse outcome attributable to tPA was 126.

3. Hanss M, Collen D. Secretion of tissue-type plasminogen activator and plasminogen activator inhibitor by cultured human endothelial cells: Modulation by thrombin, endotoxin, and histamine. J Lab Clin Med. 1987;109:97-104.

4. Hoylaerts M, Rijken DC, Lijnen HR, et al. Kinetics of the activation of plasminogen by human tissue plasminogen activator. Role of fibrin. J Biol Chem. 1982;257:2912-9.

5. Sussman BJ, Fitch TSP. Thrombolysis with fibrinolysin in cerebral arterial occlusion. J Am Med Assoc. 1958; $167: 1705$.

6. Brott T. Thrombolytic therapy for stroke. Cerebrovascular Brain Metabol Rev. 1991;3:91.

7. Sloan MA. Thrombolysis and stroke: past and future. Arch Neurol. 1987;44:748.

8. del Zoppo G. Thrombolytic therapy in cerebrovascular disease. Curr Concepts Cerebrovasc Dis. 1988;23:7.

9. del Zoppo G, Poeck K, Pessin MS, et al. Recombinant tissue plasminogen activator in acute thrombotic and embolic stroke. Ann Neurol. 1992;32:78.

10. The national institute of neurological disorders and stroke rt-pa stroke study group. Tissue plasminogen activator for acute ischemic stroke. N Engl J Med. 1995;333:1581-7.

11. Saver JL. Number needed to treat estimates incorporating effects over the entire range of clinical outcomes: novel derivation method and application to thrombolytic therapy for acute stroke. Arch Neurol. 2004;61:1066-70.

12. Hacke W, Kaste M, Bluhmki E, et al. Thrombolysis with alteplase 3 to 4.5 hours after acute ischemic stroke. N Engl J Med. 2008;359:1317-29. 
13. Lees KR, Bluhmki E, von Kummer R, et al. Time to treatment with intravenous alteplase and outcome in stroke: an updated pooled analysis of ecass, atlantis, ninds, and epithet trials. Lancet. 375:1695-1703

14. American Academy of Emergency Medicine. tPA for stroke-potential benefit, risk and alternatives. http://aaem.org/education/tpaedtool-AAEM.pdf.

15. Katzan I, Sila C, Furlan A. Community use of intravenous tissue plasminogen activator for acute stroke. Stroke. 2001;32:861.

16. Katzan I, Hammer M, Furlan A. Quality improvement and tissue-type plasminogen activator for acute ischemic stroke. A cleveland update. Stroke. 2003;34:799.

17.• Liang BA, Zivin JA. Empirical characteristics of litigation involving tissue plasminogen activator and ischemic stroke. Ann Emerg Med. 2008;52:160-4. This article summarizes trials that reach a verdict in cases where tPA is either used or not used in stroke victims. In $88 \%$ of verdicts, injury was claimed from failure of treatment with tPA.
18. The Four Elements of Medical Malpractice. Yale New Haven Medical Center: Issues in Risk Management. 1997.

19. Weintraub MI. Thrombolysis (tissue plasminogen activator) in stroke: a medicolegal quagmire. Stroke. 2006;37:1917-22.

20. $\quad$ Liang BA, Lew R, Zivin JA. Review of tissue plasminogen activator, ischemic stroke, and potential legal issues. Arch Neurol. 2008;65:1429-33.

This review showed that despite its efficacy, tPA is underutilized. The authors argue that malpractice may occur from not using tPA under the rule of negligence.

21. Hodson JD. Medical malpractice: loss of chance causality. 54 ALR 4th: 2007.

22. Saroyan $Z$. The current injustice of the loss of chance doctrine: an argument for a new approach to damages. 33 Cumb L Rev. 2002;15:99-100.

23. Thiess DE, Sattin JA, Larriviere DG. Hot topics in risk management in neurologic practice. Neurol Clin. 2010;28:429-39. 